treated with varying results. The treatment I adopted besides meeting any general indications, obtaining the best hygienic surroundings possible, and advising a strictly vegetable diet with milk, was to give twenty grains of the bromide of strontium with 5 to 10 grains of the bromide of ammonium or sodium night and morning, largely diluted with water. The strontium I increased to one drachm twice a day rapidly when I found the smaller doses did not control the attacks, and where the patient did not complain of it. I found the majority of the patients took the strontium without any depression, but generally with the production of the acne rash on the face. Liquor arsenicalis added to the mixture controlled the rash and increased the appetite. This course in all the cases materially lessened the number of the attacks and in eight of the cases there has been so far no return of them; that is after an interval of sixteen, twelve, eleven, nine, eight, eight and a balf, five and a half, and four months respectively. I cannot of course speak of the future and ultimate results of these cases, but the present improvement is so far encouraging.

Dublin.

\section{TWO CASES OF RUPTURE OF INTESTINE CAUSED BY THE SEPARATION OF ADHESIONS TO DISEASED OVARIES.}

By JOHN D. MALCOLM, M.B., C.M., F.R.C.S. Edin., SURGEON TO THE SAMARITAN FREE HOSPITAL.

THE first of these cases is the only one in my practice in which death has resulted from the removal of an inflamed orary or of any disease of the Fallopian tube. ${ }^{2}$

CASE 1.-The patient, a single woman thirty-nine years of age, had suffered from pain in the region of the left kidney for more than ten years. Occasionally the pain had been severe, but sometimes it had ceased for months together. Otherwise she had been healthy and fairly strong ti 1 October, 1893, when she was suddenly seized with a very acute pain in the right groin, and this had continued to come and go till her admission to hospital on Jan. 27th, 1894. In November, 1893, she had passed four small stones per urethram in one day without any discomfort. Menstruation had been regular except in December, 1893, when she missed a period. On admission the patient's abdomen was much distended and the tympanites was only partially relieved by laxatives and enemata. The left kidney was easily felt and was very tender to palpation; the right was felt with difficulty and was not tender. In the right lower portion of the abdomen the patient very frequently suffered from severe forcing pains. When these came on, by placing the hand on the abdominal wall one could feel a part of the bowel in the right groin swell up and become tense and hard. It would continue so for from a few seconds to half a minute, after which a gurgle could be felt and heard, the bowel would become soft, and the pain would cease. The patient declared that for some weeks the intervals between these pains had never exceeded an hour. This seemed to be the case after her admission to hospital, and I repeatedly noted a similar succession of phenomena to recur at intervals of a few minutes. Laxatives moved the bowels without appearing to make any difference to the pains. On vaginal examination it was impossible to define the uterus or neighbouring organs, which seemed all matted together and were tender to manipulation. There was no stone in the bladder. The urine was acid and of specific gravity 1012 to 1014 ; it contained a trace of albumin and a few pus and renal epithelial cells. The patient was very thin and weak, with a pulse varying from 108 to 120 and a temperature ranging between $98^{\circ} 2^{\circ} \mathrm{F}$. and $1002^{\circ}$. My opinion was that she had a calculus, or perhaps several, in her left kidney, and that the condition in the right groin was dne to some other cause, possibly to the irritation of a calculus in one of the ureters. I opened the abdomen in the middle line below the umbilicus on Feb. 5th, 1894. The cæcum and about eighteen inches of the lower end of the

1 Since this was written I have had a second fatal case. Its history will be published in a paper postponed from the last session of the Medical Society. ileum were firmly adherent together and to the pelvic peri toneum and were set free with much difficulty. When this was done I had exposed the left ovary, which was about four times its normal size and lay high up in the pelvis, well above the uterus, which was retroverted. The ovary bad been completely covered by adherent borel and was still deeply embedded in adhesions. In separating it I ruptured several cysts, letting out a thick, tar-like fluid (altered blood) in considerable quantity. When the ovary and Fallopian tube had been removed I found that the latter was occluded at its fibrinated extremity. I could feel the right ovary low down in Douglas's pouch at the end of a narrow space between firm adhesions. As it seemed to be enlarged and likely to be a source of irritation I removed it, but in order to do so I had to separate the uterus from the back of Douglas's pouch, to which it was firmly adherent. The ovary was, in fact, slightly enlarged and, like its fellow, it contained cysts (altered follicles) full of tar-like material. The right tube was fairly healthy. There were still some strong adhesions of the small intestine to the sigmoid flexure and posterior wall of the atdomen, which I thought it advisable to separate. When the bowel was brought freely out of the abdomen I found that its walls were much damaged at various places, the divided adhesion at several points being almost as hard as cartilage. There was no perforation of the gut, but 1 thought it best to draw the peritoneum together by sutures over some parts of its walls that looked weak. These proceedings had occn yied rearly three hours, and I considered it undesirable to begin another difficult piece of manipulation such as an attempt to ubstract a stone or stones from the kidney would have necessitated. I therefore fixed a drainage-tube in the pelvis and closed the wound, hoping to be able to deal with the renal tronble on a future occasion. The patient was put to bed at 12.30 R.M. in a very collapsed state, with a feeble pulse beating about 140 to the minute and a temperature of $996^{\circ}$. The temperature slowly rose to $100.4^{\circ}$ at 9 P.M., the pulse gradually having fallen by that hour to 108 , but still being very feeble. At 1030 P.M. three ounces of red $f$ laid, free from odour, had collected in the sponge over the open end of the drainagetube. The abdomen was soft and flat and the patient's facial expression was good. Flatus had passed freely from the bowel each time a tube had been passed into the rectum at intervals of three hours. Eleven ounces of urine were secrefed in the first eleven hours after the operation. From 9 P.M. on the day of the operation till $2 \mathrm{~A}$.M. the temperature was steady at $100.4^{\circ}$, and at the end of that time the pulse had slowed down to 100 per minute. Then the patient complained of severe pain in the abdomen, which did not last long; but after it she was very restless and her rate of breathing became rapid. At 3 A.M., when I saw her, the temperature had risen to $101 \cdot 4^{\circ}$ in the vagina and the pulse remained 100 , but the whole aspect of the patient had changed and it was evident that something serious had occurred. It was casy to surmise that the bowel had ruptured, but the evidence to this affect was not quite conclusive, and if it had been the feebleness of the patient would have precluded all hope of gaining any advantage by re-opening the abdomen. At 9.30 A.M. about an ounce of clear, red, odourless fluid had escaped from the drainage-tube. The temperature was $101 \cdot 4^{\circ}$, the pulse was 100 , and very little urine had been secreted during the night. Flatus had continued to pass freely from the bowel when a rectal tube was inserted and the abdomen was soft and tlat. After this time the pulse quickened, and the patient gradually sank and died twenty-eight hours after the operation, the temperature towards the end falling to $101^{\circ}$. At the necropsy it was found that the intestine had given way at one of the points where an old adhesion had been separated. There were several ounces of purulent fluid mixed with frces in the peritoneal cavity, and there was an early stage of general peritonitis. The ureters were normal There was a large calculus of irregular shape in the pelvis of the left kidney and there was also a gall-stone in the gallbladder.

In ordinary cases of this kind I prefer to invert and support by sutures every part of the gut that appears seriously damaged. There were, however, in this case several places where the divided adhesions and the wall of the intestine were so hard and inelastic that it would scarcely have been possible to invert the injured parts, which, moreover, seemed to be strong enough to resist any pressure from within the bowel. If I had attempted to sew over these portions the sutures would probably have cut themselves out of the 
tissues on being tightened, and I shoulc have had to resect a large piece of intestine or several small pieces-a proceeding which the patient was not in a state to undergo with any hope of success. In any future case resembling this one I should be content with removing diseased parts, separating bowel adhesions as little as possible, and trusting to the coils of the intestines being able to arrange themselves so as to admit of a free passage of their contents downwards. I have generally adhered to this resolution, but I broke it in the following case.

CAsE 2.-A married woman forty-eight years of age had noticed her abdomen to be swollen in March, 1882. Mr. Knowsley Thornton had operated on her, removing a left ovarian tumour, on June 7 th of the same year, and a good recovery had followed. Shortly before Christmas, 1894, the patient felt a burning pain in the right groin, became very depressed, especially when tired, and began to lose flesh. In October, 1895, Dr. Homan of Tichfield advised her to consult Mr. Thornton. She had then a small tumour in the right side of the pelvis, for the ręmoval of which Mr. Thornton aent her to the Samaritan Free Hospital under my care I opened the abdomen on Nov. 19th, 1895, and I found a tumour about the size of an orange deeply embedded in the right broad ligament, with coils of small intestine firmly adherent to its posterior surface. It was not possible to bring the tumour forward, and I had to separate the intestine by touch until I could reach and tie the broad ligament below the growth. I had to tie the broad ligament in several piecea, cutting the tumour away as I secured its attachments. When the tumour was removed I found that it was still impossible to bring the intestines into view where their adhesions had been separated, and in order to see what damage had been done I thought it better completely to separate the gut from its adhesions lower in the pelvis. When this had been done I brought out of the abdomen a mass of intestine twisted into several coils which were adherent to each other at many points. I carefully separated the adbesions, which were so close that it was sometimes difficult to tell in which direction the lumen of the gut lay. When all were released there were several places where the muscular cat of the bowel was torn more or less, and at one point there was a perforation of the mucous coat with escape of fxces. 'This last opening was carefully closed by Lembert's sutures, and the more seriously damaged places were also inverted and supported by sutures. When all had been made secure and the parts had been carefully cleansed I closed the abdomen without using a drainage tube. The patient was put to bed after a long operation in a state of extreme collapse, and it was some hours before she showed signs of reviving. Recovery was otherwise satisfactory; the highest temperature in the vagina was $100 \cdot 8^{\circ}$, the highest pulse was 114 , and both temperature and pulse quickly fell. The bowels were moved by enema on the fifth day and they gave no trouble.

An unusual accident occurred in Case 2. I noted that I had removed the last suture from the wound on the eleventh day after the operation; but nine days later the wound did not seem to be well healed at one point. It very nearly healed over, however, and the patient went home a month after the operation. Two months later Dr. Homan wrote that the patient was very well, but that there was a small bit of the wound still unhealed with a piece of silk protruding which he could not pull away. A week later anotber end of silik was seen, a knot was discovered, and the suture was removed.' I had not used any deep sutures, so I presume this suture must have been rather tiglit and have cut the skin and so buried itself. It had then caused very little irritation until the patient began to get about freely. I saw hor in July. 'The wound was then completely healed, there was no difficulty with the bowels, the patient's condition was very satisfactory, and she was gaining strength.

As in these cases it is often difficult to decide whether to separate old adhesions or to leave them alone, it should be borne in mind that adhesions in themselves are comparatively harmless, and that it is only when they fix coils of intestine or other viscera in abnormal positions that they cause trouble or danger. Hence strong adhesions of intestine that are known to have existed without having caused obstruction of, or difficulty with, the bowels are as a rule best left alone. If, however, it has been necessary to separate adhesions of neighbouring coils it may be that the released coils by assuming new positions will cause an obstruction of the gut. Under such circumstances, as in the two cases recorded, it may be wiser completely to separate all adhesions. In the first case related above the adhesions were undoubtedly causing difficulty to the peristaltic action of the bowels, and it was not possible to tell at which point the obstruction lay, or whether the removal of the ovaries without further separation of adhesions would suffice to effect a cure. Moreover, in both the cases recorded I was compelled to separate adhesions to a certain extent, and it was impossible to find out exactly what damage had been done without separating more adhesions, so that the risks of going on with the freeing of the intestines seemed less than those of leaving them partially released. In the second case, although the adhesions were.strong and close, there were none of those very hard adhesions which were found in the first case and which I have described as of cartilaginous consistence. This made the conditions much more easy to deal with, and the second patient had also the advantage of being in fairly good health at the time of the operation, whereas the first one was worn to a shadow and extremely feeble from constantly recurring attacks of pain.

Portman-street, W.

\section{$\triangle$ CASE OF FOOT AND MOUTH DISEASE IN THE HUMAN SUBJECT.}

BY JOHN GLAISTER, M.D., F.F.P.S.GLASG., D.P.H. CAMB., \&C.,

PROFESSOR OF FOREXSIC MEDICINE AND PUBLIC HEALTH, ST. MCNGO'S COLIEGE, GLASGOW.

Is riew of the increased attention which is being paid in these days to the diseases of the lower animals which are communicable to man the following notes respecting an attack of the above disease in the human subject will not prove uninteresting to the readers of THE LANCET. Such cases are comparatively rare, and by reason of their rarity ought to be carefully recorded when encountered. The literature, too, of the affection is by no means copious, although it is sufficiently large to prove what is now unquestioned-that it is a disease which under certain conditions is communicable from the lower animals to man, while it leaves open to doubt its communicability from man to man. The facts of the case which came under my observation were as follows.

In December of last year I was asked to see a woman aged forty-four years. She was a person who usually enjoyed vigorous health. Her symptoms of illness at that visit were the following: She had a history of a rigor, a febrile condition (temperature $102^{\circ} \mathrm{F}$.), a dry, furred tongue, anorexia, considerable thirst, and a general feeling of soreness of the muscles of the trunk and limbs. The room in which she lay was darkened on account of conjunctivitis of both eyes causing her to be unable to bear the light. In addition, she suffered from coryza to a slight degree and from a trifling cough. On the face and neck there was the faintest appearance of a rash, which looked not unlike the initial stage of the eruption of measles. In short, all her symptoms pointed to an attack of ordinary measles, which, however, she assured me she had had upon a former occasion. Next day the conjunctivitis had considerably increased and the conjunctivæ were beginning to assume a tumid appearance ; the coryza also had increased and the nasal mucous membrane was also somewhat swollen. She complained of tenderness upon micturition. The appearance of rash on the face and neck had now nearly cleared off. The temperature was now $102 \cdot 6^{\circ}$. She had had a sleepless night, and she was very nervous. The most prominent symptom observable at this visit, and one of which she had not formerly complained, was a painful condition of the mouth, of which upon examination the whole of the mucous membrane was found to be in an inflamed and tumified state. It had just the appearance as if it had been painted over with blistering fluid or as if she had washed her mouth with strong carbolic acid. This inflamed condition extended over the lips to the margin of the skin. In consequence of the tongue having shared the same inflamed and swollen state her articulation was difficult and thick, and the act of swallowing fluid had become both painful and difficult. A rash had now begun to show itself on the forearms, which consisted of circular and oral spots slightly raised above the level of the skin 